\title{
Systemic Acquired Resistance-Mediated Control of Pine Wilt Disease by Foliar Application With Methyl Salicylate
}

\begin{abstract}
Hee Won Jeon ${ }^{1}$, Ae Ran Park', Minjeong Sung ${ }^{1}$, Namgyu Kim², Mohamed Mannaa ${ }^{2}$, Gil Han², Junheon Kim³, Yeonjong Koo', Young-Su Seo ${ }^{2}$ and Jin-Cheol Kim ${ }^{1 *}$
\end{abstract}

\begin{abstract}
1 Department of Agricultural Chemistry, College of Agriculture and Life Sciences, Institute of Environmentally Friendly Agriculture, Chonnam National University, Gwangju, South Korea, ${ }^{2}$ Department of Integrated Biological Science, College of Natural Science, Pusan National University, Busan, South Korea, ${ }^{3}$ Forest Insect Pests and Diseases Division, National Institute of Forest Science, Seoul, South Korea
\end{abstract}

Pine wilt disease (PWD), caused by the pinewood nematode, is the most destructive disease in pine forest ecosystems worldwide. Extensive research has been done on PWD, but effective disease management is yet to be devised. Generally, plants can resist pathogen attack via a combination of constitutive and inducible defenses. Systemic acquired resistance (SAR) is an inducible defense that occurs by the localized infection of pathogens or treatment with elicitors. To manage PWD by SAR in pine trees, we tested previously known 12 SAR elicitors. Among them, methyl salicylate (MeSA) was found to induce resistance against PWD in Pinus densiflora seedlings. In addition, the foliar applications of the dispersible concentrate-type formulation of MeSA (MeSA $20 \mathrm{DC})$ and the emulsifiable concentrate-type formulation of MeSA (MeSA 20 EC) resulted in significantly reduced PWD in pine seedlings. In the field test using 10-yearold P. densiflora trees, MeSA 20 DC showed a 60\% decrease in the development of PWD. Also, MeSA 20 EC gave the best results when applied at $0.1 \mathrm{mM}$ concentration 2 and 1 weeks before pinewood nematode (PWN) inoculation in pine seedlings. qRTPCR analysis confirmed that MeSA induced the expression of defense-related genes, indicating that MeSA can inhibit and delay the migration and reproduction of $P W N$ in pine seedlings by modulating gene expression. These results suggest that foliar application of MeSA could reduce PWD incidence by inducing resistance and provide an economically feasible alternative to trunk-injection agents for PWD management.

Keywords: pine wilt disease, methyl salicylate, systemic acquired resistance, foliar application, qRT-PCR

\section{INTRODUCTION}

Pine wilt disease (PWD), caused by the pinewood nematode (PWN), Bursaphelenchus xylophilus, is the most destructive disease in pine forest ecosystems worldwide. PWN can easily migrate within infected trees, but because they cannot move between hosts, they are transmitted through the vector Monochamus spp. (Mamiya, 1988; Kim et al., 2021). PWN is fatal to healthy susceptible pine trees, such as Pinus densiflora, $P$. thunbergii, and $P$. koraiensis and new susceptible species are being discovered (Yang and Wang, 1989; Li et al., 2020). PWD-infected pine trees initially turn their needles yellowish to reddish-brown, and then subsequently wilt and die within a few months. PWD 
was first reported in Japan in 1905 (Yano, 1913), after which it gradually reached Europe (Portugal and Spain) as well as East Asian countries such as China, Taiwan, and Korea (Cheng et al., 1983; Tzean and Jan, 1985; Yi et al., 1989; Mota et al., 1999; Abelleira et al., 2011).

The serious threat posed by PWN has led to considerable efforts to control the disease. Disease control mostly relies on use of chemicals such as metam sodium for fumigation of infected trees, aerial spraying of pesticides to control pine sawyer, and trunk injection of abamectin and emamectin benzoate (Kwon et al., 2005; Lee et al., 2009; Korea Forest Service, 2013). However, with the growing awareness regarding environmental conservation, there is now a demand for environmental-friendly control measures. In line with this, research on elucidating resistance mechanisms related to the relationship between pathogens and host pines is being emphasized upon.

Plants can resist pathogen attack through a combination of constitutive and inducible defense mechanisms, which suppress pathogen reproduction and spread (Spoel and Dong, 2012; Vlot et al., 2021). Induced resistance has two forms: systemic acquired resistance (SAR) and induced systemic resistance (ISR). Specifically, SAR is known as long-lasting and broad-spectrum resistance that sets in following a localized infection by a variety of pathogens or treatment with SAR elicitors. The activation of SAR requires transfer of signals from the primarily infected tissue to systemic tissue. Various substances that activate SAR, such as $\beta$-aminobutyric acid (BABA), methyl salicylate (MeSA), and azelaic acid (AZA) have recently been reported (Park et al., 2007; Jung et al., 2009; Ji et al., 2015).

MeSA is a naturally occurring compound that is widely distributed in many plants and is classified into a group of plant hormones that play various regulatory roles in plant metabolism (Hayat and Ahmad, 2007). MeSA, a long-distance signaling compound of SAR, is volatilized and transported to the air. Airborne MeSA is absorbed by uninfected distal tissues, converted into SA, after which it eventually activates SAR (Dempsey and Klessig, 2012). Recently, upon application of MeSA, development of disease resistance was reported against bacterial blight caused by Xanthomonas oryzae pv. oryzae in rice and gray mold caused by Botrytis cinerea in grape (Kalaivani et al., 2016; García-Pastor et al., 2020).

It has been demonstrated that MeSA can effectively control PWD by inducing flavonoid biosynthesis through comparative in vivo transcriptomics on pine seedlings (Park et al., 2020). However, the establishment of optimal treatment conditions for high efficiency of MeSA and application to the field have not been reported. The purpose of this study was to establish the optimal formulation and processing conditions of MeSA for effectively controlling PWD through in vivo assays and field experiments, and to elucidate the mechanisms underlying the effects of MeSA against PWD based on qRT-PCR analysis.

\section{MATERIALS AND METHODS}

\section{Chemicals and Formulations}

AZA (>98\%), azoxystrobin (98.4\%), BABA (>98\%), $\gamma$-aminobutyric acid (GABA, > 99\%), glucan (from black yeast), isotianil (ITL, 98.2\%), methyl jasmonate (>90\%), MeSA (>99\%), probenazole (PBZ, 98.5\%), salicylic acid (SA, > 99.5\%), tiadinil (TDL, 98.9\%), and validamycin A (VMA, 64.24\%) were purchased from Tokyo Chemical Industry Co., Ltd. (Tokyo, Japan). The four elicitors selected following screening tests (GABA, MeSA, PBZ, and SA) were formulated by Yoosung Chemical R\&T Co., Ltd. (Daejeon, South Korea). A soluble concentrate-type formulation of GABA (GABA 20 SL) was prepared by mixing $20 \%(\mathrm{w} / \mathrm{w})$ GABA, $10 \%(\mathrm{w} / \mathrm{w})$ polyethylene glycol-400, 5.0\% (w/w) sodium lignin sulfonate, $3.0 \%(\mathrm{w} / \mathrm{w})$ monotridecyl ether (POE9), and 62.0\% (w/w) water. A dispersible concentrate-type formulation of MeSA (MeSA 20 DC) was prepared by mixing 20\% (w/w) MeSA, $30 \%(\mathrm{w} / \mathrm{w})$ propylene glycol monomethyl ether, $20 \%(\mathrm{w} / \mathrm{w})$ propylene glycol, and 20\% (w/w) CR-FL3PG (the mixture of triethanolamine, and polyoxyethylene tristyrylphenyl ether phosphate). An emulsifiable concentrate-type formulation of MeSA (MeSA 20 EC) was prepared by mixing 20\% (w/w) MeSA, $20 \%(\mathrm{w} / \mathrm{w})$ propylene glycol monomethyl ether, 20\% (w/w) CR-MOC25 (the mixture of ethoxylated castor oil, calcium dodecylbenzenesulfonate, and tristyrylphenol ethoxylates) as an emulsifier, and 40\% (w/w) methylated soybean oil (MOS2). A suspension concentrate-type formulation of PBZ (PBZ 20 SC) was prepared by mixing $20 \%(\mathrm{w} / \mathrm{w}) \mathrm{PBZ}, 8.0 \%(\mathrm{w} / \mathrm{w})$ propylene glycol, 5.0\% (w/w) CR-FL3PG, $0.12 \%(\mathrm{w} / \mathrm{w})$ xanthan gum, $0.1 \%$ (w/w) silicon defoamer, and 66.78\% (w/w) water. A soluble concentrate-type formulation of SA (SA $20 \mathrm{SL}$ ) was prepared by mixing 20\% (w/w) SA, $11.6 \%$ (w/w) $\mathrm{NaOH}(50 \%), 10 \%$ (w/w) polyethylene glycol-400, 5.0\% (w/w) sodium lignin sulfonate, $3.0 \%(\mathrm{w} / \mathrm{w})$ monotridecyl ether (POE9), and 50.4\% (w/w) water.

\section{Nematodes and Plants}

The PWN Bursaphelenchus xylophilus, which causes PWD, was isolated from infected pine trees and obtained from the National Institute of Forest Research (NIFoS; Seoul, South Korea). PWN was cultured on the mycelia of Botrytis cinerea on potato dextrose agar (Difco; Becton, Dickinson and Company, Sparks, $\mathrm{MD}$, United States) at $25^{\circ} \mathrm{C}$ for 1 week in dark (Maehara and Futai, 2000). PWNs were harvested from the medium using the Baermann funnel method (Baermann, 1917). For the in vivo experiment, 3-year-old Pinus densiflora and $P$. thunbergii seedlings of $40-50 \mathrm{~cm}$ height and $0.5-1.0 \mathrm{~cm}$ diameter were obtained from Daelim seedling farm (Okcheon, South Korea) and transplanted into $15-\mathrm{cm}$ diameter pots containing sterilized nursery soil in the greenhouse, which was maintained at an average temperature of $25^{\circ} \mathrm{C}$ with the relative humidity of $70 \%$.

\section{Screening of Various Elicitors Against Pine Wilt Disease by Means of Trunk Injection}

Ten chemicals (except glucan and ITL) were dissolved in an aqueous solution containing $10 \%$ methanol, while glucan and ITL were dissolved in an aqueous solution containing $10 \%$ dimethylformamide, at concentrations of 200 and $20 \mu \mathrm{g} / \mathrm{mL}$. Three-year-old $P$. densiflora seedlings were treated with $100 \mu \mathrm{L}$ of each solution via trunk injection. A hole was drilled using a $1.8 \mathrm{~mm}$ drill bit at a $45^{\circ}$ angle in the trunk of a pine seedling, 
$5 \mathrm{~cm}$ above the ground. A $200 \mu \mathrm{L}$ pipette tip (Axygen Scientific Inc., Union, CA, United States) was inserted into the hole. One hundred microliters of each chemical solution was injected into the pipette tip. The pipette tip was covered with Parafilm ${ }^{\circledR} \mathrm{M}$ (Heathrow Scientific, Vernon Hills, IL, United States) to prevent drying, following which a small hole was made in it using a pin. One week later, the treated pine seedlings were inoculated with PWN, as described below. After making a small slit with a surface-sterilized knife, a small piece of absorbent cotton was inserted into the slit, and a water suspension of nematodes $(2,000$ nematodes in $100 \mu \mathrm{L}$ ) was pipetted onto the absorbent cotton. The slits were then covered with Parafilm ${ }^{\circledR}$ to prevent drying (Kwon et al., 2010). Distilled water containing 10\% methanol was used as an untreated control. Three replicates were included in each treatment, and each experiment was performed twice.

\section{Effects of Four Selected Elicitors Formulations Against Pine Wilt Disease Upon Foliar Application}

Formulations of GABA 20 SL, MeSA 20 DC, PBZ 20 SC, and SA 20 SL were dissolved in distilled water at a concentration of $10 \mathrm{mM}$ each, and then $5 \mathrm{~mL}$ of each solution was foliar sprayed onto 3 -year-old $P$. densiflora seedlings. The treatment was conducted twice at an interval of 1 week. One week after the second treatment, the treated 3 -year-old pine seedlings were inoculated with 2,000 PWNs. Distilled water containing 250 $\mu \mathrm{g} / \mathrm{mL}$ Tween ${ }^{\circledR} 20$ was used as an untreated control and five replicates were used. The severity of PWD was assessed according to the wilting and the ration of the brown leaves of the whole plant leaves (Proença et al., 2010; Kim et al., 2019). To evaluate the control value of treatment against B. xylophilus, control value was measured using below formula (Kwon et al., 2010), control value $(\%)=(1$-disaease severity of treatment/disease severity of untreated control) $\times 100$.

\section{In vitro Nematicidal Activity of Methyl Salicylate}

To confirm whether the effect of MeSA against PWD was caused by direct nematicidal activity, the nematicidal activity of MeSA was evaluated on B. xylophilus. After the suspension containing approximately 50 nematodes was added to each well of a 96-well microplate (Becton Dickinson, Franklin Lakes, NJ, United States), treatments with three concentrations of MeSA, 1,000, 333, and $111 \mu \mathrm{g} / \mathrm{mL}$, were carried out. Sterilized distilled water was used as the negative control. The plates were gently shaken and incubated in a dark plastic box with $100 \%$ humidity at an average temperature of $25^{\circ} \mathrm{C}$. All experiments were conducted in triplicates and repeated twice. Nematicidal activity was evaluated at 3 days post-treatment, under an optical microscope (Leica DM IL LED; Leica Microsystems CMS GmbH, Wetzlar, Germany). To analyze the nematicidal activity of MeSA against B. xylophilus, mortality was converted to percentage mortality and corrected using Abbott's formula (Abbott, 1925), Mortality $(\%)=[($ Mortality percentage in treatment - Mortality percentage in the negative control $) /(100-$ Mortality percentage in the negative control)] $\times 100$.

\section{Evaluation of Disease Control Efficacy of Methyl Salicylate 20 DC Against Pine Wilt Disease Under Field Conditions}

The field experiment was performed in a pine forest located in Seongbang-ri, Gonmyeong-myeon, Sacheon-si, Gyeongsangnam-do province, Republic of Korea, in 2017. Ten-year-old $P$. densiflora trees, $3-5 \mathrm{~m}$ in height and $8-10 \mathrm{~cm}$ in diameter at chest height, were used for the experiment. MeSA 20 DC formulation was prepared in distilled water at a concentration of $10 \mathrm{mM}$ and $500-800 \mathrm{~mL}$ for a tree. This solution was foliar sprayed onto 10-year-old $P$. densiflora trees through Sprayer Clover (Model TH-33; Taehwan Co., South Korea). The treatment was conducted twice at an interval of 1 month. One month after the second treatment, the treated pine trees were inoculated with PWN via trunk injection. A hole was drilled using a $10 \mathrm{~mm}$ drill bit at a $45^{\circ}$ angle into the trunk of a pine tree, $30 \mathrm{~cm}$ above the ground. One milliliter of water suspension of nematodes (10,000 nematodes) was pipetted into the hole. Inoculation of PWN was performed on June 2, 2017, and the average temperature was $20^{\circ} \mathrm{C}$. After inoculation with $\mathrm{PWN}$, the hole was closed using a cork stopper. Distilled water containing $250 \mu \mathrm{g} / \mathrm{mL}$ Tween ${ }^{\circledR} 20$ was foliar sprayed as a negative control, while emamectin benzoate 2.15 EC (Affirm, Syngenta Co., Seoul, Korea) was administered via trunk injection as a positive control. Sixteen trees were used in each treatment. The incidence of PWD was assessed in terms of the trees being either alive or dead.

\section{Selection of Optimal Formulation of Methyl Salicylate Against Pine Wilt Disease in 3-Year-Old Pinus densiflora and Pinus thunbergii Seedlings}

MeSA 20 DC and MeSA 20 EC were formulated by Yoosung Chemical R\&T Co., Ltd. To select the optimal formulation of MeSA, in vivo assays were performed on $P$. densiflora and $P$. thunbergii seedlings. Each sample was dissolved in distilled water at a concentration of $10 \mathrm{mM}$, and then $5 \mathrm{~mL}$ of these solutions were foliar sprayed onto 3-year-old $P$. densiflora and $P$. thunbergii seedlings. The treatment was conducted twice at an interval of 1 week. One week after the second treatment, the treated seedlings were inoculated with 2,000 PWNs. Distilled water containing $250 \mu \mathrm{g} / \mathrm{mL}$ Tween ${ }^{\circledR} 20$ was used as a negative control, and emamectin benzoate $20 \mathrm{mg} / \mathrm{mL}$ was used as a positive control. Five replicates were included in each treatment group. The severity of PWD was assessed according to the wilting and discolored area of the needles.

\section{Determination of Optimal Concentration and Application Time of Methyl Salicylate 20 EC Against Pine Wilt Disease in 3-Year-Old Pinus densiflora and Pinus thunbergii Seedlings}

To determine the optimal concentration of MeSA 20 EC, in vivo assays were performed at various concentrations of MeSA 20 EC to select the optimal formulation for MeSA. MeSA 20 EC was dissolved in distilled water at concentrations of $10,0.1$, and 
$0.01 \mathrm{mM}$ and then $5 \mathrm{~mL}$ of these solutions were foliar sprayed onto 3-year-old $P$. densiflora and $P$. thunbergii seedlings. The treatment was conducted twice at an interval of 1 week.

For determination of optimal application time for MeSA 20 $\mathrm{EC}$, it was dissolved in distilled water at a concentration of $0.1 \mathrm{mM}$, which was selected as the optimal concentration, and then foliar sprayed as follows: 1 week before inoculation (WBI) with PWN; 2 and 1 WBI with PWN; 3 and 1 WBI with PWN; 4 and 1 WBI with PWN. In both the experiments aimed at optimization of concentration and application time, the treated seedlings were inoculated with 2,000 PWNs 1 week after the second treatment. Distilled water containing $250 \mu \mathrm{g} / \mathrm{mL}$ Tween ${ }^{\circledR}$ 20 was used as a negative control. Five replicates were included in each treatment group. The severity of PWD was assessed according to the wilting and discolored area of the needles.

\section{Assessment of the Effect of Methyl Salicylate on the Expression of Defense-Related Genes Using qRT-PCR}

Three-year-old $P$. densiflora seedlings were used to analyze the effect of MeSA on expression of defense-related genes. The seedlings were foliar sprayed with MeSA $20 \mathrm{EC}(0.1 \mathrm{mM})$ at an interval of 1 week. For the untreated controls, distilled water containing Tween ${ }^{\circledR} 20(250 \mathrm{mg} / \mathrm{L})$ was used. One week after the second treatment, the treated pine seedlings were inoculated with PWN (2,000 nematodes/100 $\mu \mathrm{L})$. Three replicates were included in each treatment.

Total RNA was extracted from the pine needles using a hexadecyltrimethylammonium bromide (CTAB)-based extraction buffer (Azevedo et al., 2003). Sampling was performed at 1, 3, and 7 days post-first treatment; 1,3 , and 7 days postsecond treatment; and 1, 3, and 7 days post-inoculation with PWN, to confirm the change over time. First, the samples were coarsely ground in liquid nitrogen using a mortar and pestle. The coarsely ground sample $(0.5 \mathrm{~g})$ was put into a $2 \mathrm{~mL}$ e-tube and completely ground using a tissuelyser (Qiagen Inc., Valencia, CA, United States). The ground sample was mixed with $1 \mathrm{~mL}$ extraction buffer containing $0.1 \mathrm{M}$ Tris- $\mathrm{HCl}(\mathrm{pH}$ 8.0), $30 \mathrm{mM}$ ethylenediaminetetraacetic acid, $2 \mathrm{M} \mathrm{NaCl}, 2 \%$ CTAB, $0.05 \%$ spermidine, $2 \%$ polyvinylpolypyrrolidone, $2 \% 2$ mercaptoethanol, and $1.5 \mathrm{mg} / \mathrm{mL}$ proteinase $\mathrm{K}$. The suspension was incubated at $42^{\circ} \mathrm{C}$ for 90 min and then extracted with $15 \mathrm{~mL}$ chloroform-isoamyl alcohol (24:1). Total RNA was precipitated using $2 \mathrm{M} \mathrm{LiCl}$. Total RNA was further purified using the IQeasy Plus Plant RNA Extraction Mini Kit (iNtRON, Seongnam, South Korea). RNA quality was assessed using a NanoDrop ND1000 spectrophotometer (NanoDrop Technologies, Wilmington, DE, United States). The cDNA library was synthesized using oligo (Ji et al., 2015) primers and SuperScript IV reverse transcriptase (Invitrogen Inc., Carlsbad, CA, United States), following the manufacturer's guidelines. The PCR primers used in this study (Table 1; Hirao et al., 2012; Lee et al., 2019) were synthesized by Genotech (Daejeon, South Korea).

qRT-PCR was performed using $\mathrm{iQ}^{\mathrm{TM}}$ SYBR Green supermix with a CFX96 Touch ${ }^{\text {TM }}$ Real-Time PCR Detection System (BioRad, Hercules, CA, United States). Relative expression levels were
TABLE 1 | Primers used in this study.

\begin{tabular}{lcc}
\hline Gene & \multicolumn{1}{c}{ Sequence $\left(\mathbf{5}^{\prime} \rightarrow \mathbf{3}^{\prime} \mathbf{)}\right.$} & References \\
\hline PR-1 For & TGCCCCTTCAGGTAATCGT & Hirao et al., 2012 \\
PR-1 Rev & GCGGGTCGTAGTTGCAGATAA & \\
PR-2 For & CGACAACATTCGCCCCTTCT & \\
PR-2 Rev & CTGCAGCGCGGTTGAATAT & \\
PR-5 For & GAACCAGTGCCCATACACAGTCT & \\
PR-5 Rev & CCTGCGGCAACGTTAAAAGTC & \\
PR-9 For & ACACCACCGTGCTGGACATT & \\
PR-9 Rev & GTGCGGGAGTCGGTGTAGAG & \\
ETS For & CGAATGTAATCCGAAGTTCA & \\
ETS Rev & CCATCCCAAACCACCAGTCT \\
XET For & TCTGCGCCCCTACTITCC \\
XET Rev & AGCTGGGCGATTGATCATGT & \\
PdEIF4A-2 For & AATGCTTGTCCCACCAACAC & Lee et al., 2019 \\
PdEIF4A-2 Rev & AGTGTCAGGCGCTAGTTTG & \\
\hline
\end{tabular}

calculated using the comparative $2^{-\Delta \Delta C T}$ method, with EIF4A-2 as an internal control (Livak and Schmittgen, 2001).

\section{Statistical Analysis}

Statistical analysis was performed to determine significant differences between the groups. All statistical analyses were performed using SPSS statistical analysis software (version 21.0 for Windows; SPSS, IBM Corp., Armonk, NY, United States). Data were evaluated using one-way analysis of variance, and the means of the treatments were determined using the Tukey's HSD test. Results with $p<0.05$ were considered statistically significant.

\section{RESULTS}

\section{Screening of Various Elicitors Against Pine Wilt Disease by Means of Trunk Injection}

Twelve elicitors known to induce resistance in various plants were used for in vivo screening of PWD in $P$. densiflora seedlings (Table 2). Treatment with the elicitors was carried out via trunk injection to ascertain their effects. Among the 12 candidates, four elicitors (GABA, MeSA, PBZ, and SA) showed more than 75\% control values at both the concentrations of 200 and $20 \mu \mathrm{g} / \mathrm{mL}$ (Table 2). Glucan, TDL, and VMA showed control effects at specific concentrations but their effects at the other concentration were not significant.

\section{Control Effect of the Four Selected Elicitor Formulations Against Pine Wilt Disease Upon Foliar Application Onto 3-Year-Old Pinus densiflora Seedlings}

The four selected elicitors were formulated for stability according to the properties of each chemical. Foliar application was used to control PWD more effectively. Among the four selected formulations, only MeSA 20 DC significantly reduced the severity of PWD in P. densiflora seedlings. Disease severity in P. densiflora 
TABLE 2 | screening of various elicitors against pine wilt disease by trunk injection into 3-year-old Pinus densiflora seedlings.

\begin{tabular}{lcc}
\hline Treatment & \multicolumn{2}{c}{ Concentration $(\mu \mathrm{g} / \mathrm{mL})$} \\
\cline { 2 - 3 } & $\mathbf{2 0 0}$ & $\mathbf{2 0}$ \\
\hline Azelaic acid & + & + \\
Azoxystrobin & + & + \\
$\beta$-aminobutyric acid & + & - \\
$\gamma$-aminobutyric acid & +++ & ++ \\
Glucan & + & ++ \\
Isotianil & ++ & + \\
Methyl jasmonate & + & + \\
Methyl salicylate & +++ & ++ \\
Probenazole & ++ & +++ \\
Salicylic acid & +++ & ++ \\
Tiadinil & ++ & + \\
Validamycin A & ++ & - \\
Control & - & + \\
\hline
\end{tabular}

+++ , high activity, more than $75 \%$ of control value; ++ , moderate activity, $74-50 \%$ of control value; +, low activity, $49-25 \%$ of control value; -, no activity, $24-0 \%$ of control value.

seedlings foliar sprayed with MeSA 20 DC was significantly reduced compared to that in the untreated control. The MeSA 20 DC-treated and -untreated groups showed disease severity of 20 and $84 \%$, respectively, at 43 days post-inoculation with PWN, while the MeSA 20 DC-treated group showed 76.2\% control value (Figure 1). GABA 20 SL, PBZ 20 SC, and SA 20 SL did not affect PWD when foliar sprayed. Additionally, when tested against the PWN, B. xylophilus, no nematicidal activity of MeSA was observed. MeSA led to 3.5, 1.9, and $0.8 \%$ mortality at concentrations of $1,000,333$, and $111 \mu \mathrm{g} / \mathrm{mL}$, respectively, and showed results comparable to distilled water (mortality: 2.1\%) used as a negative control (Supplementary Table 1). On the contrary, emamectin benzoate, which used as a positive control, showed a high mortality of $98.8 \%$ even at a very low concentration of $0.11 \mu \mathrm{g} / \mathrm{mL}$. MeSA leads to approximately $95 \%$ mortality at a concentration of $2,000 \mu \mathrm{g} / \mathrm{mL}$ (Kim et al., 2011), but no such activity was observed when it was used at a concentration of $200 \mu \mathrm{g} / \mathrm{mL}$ in the screening test. Therefore, it indirectly suggested that the control effect of MeSA against PWD was due to inducing resistance, not direct nematicidal activity.

\section{Determination of Optimal Formulation, Concentration, and Application Time of Methyl Salicylate Against Pine Wilt Disease in 3-Year-Old Pinus densiflora and Pinus thunbergii Seedlings}

Two formulations of MeSA were evaluated for their ability to control PWD in P. densiflora and P. thunbergii seedlings, which are susceptible to PWD. In P. densiflora, the MeSA 20 DC and MeSA 20 EC formulations showed 47.7 and 52.3\% control values, respectively, compared to the untreated group; moreover, there was no significant difference between the two formulations (Figure 2). In contrast, against $P$. thunbergii, the
MeSA 20 DC and MeSA 20 EC formulations showed 6.7 and $57.8 \%$ control values, respectively, compared to the untreated group. Furthermore, it was confirmed that the activity of the MeSA 20 DC formulation was significantly reduced. Emamectin benzoate, which was used as a positive control in P. densiflora and $P$. thunbergii, showed excellent control values of 95.5 and $100 \%$, respectively. Therefore, MeSA $20 \mathrm{EC}$ was selected as the optimal formulation, and was used for the subsequent experiments.

Subsequently, the optimal concentration of MeSA 20 EC formulation was determined for the effective control of PWD. In $P$. densiflora, the control value was higher $(54.8 \%)$ at $0.1 \mathrm{mM}$ MeSA 20 EC, a concentration 100-fold lower than the other tested concentration, which was $10 \mathrm{mM}$ (33.3\%) (Figures 3A,C). However, $0.01 \mathrm{mM}$ did not control PWD. The results in $P$. thunbergii showed a higher control value at $0.1 \mathrm{mM}(56.3 \%)$ than at $10 \mathrm{mM}(45.8 \%)$, similar to the results in $P$. densiflora. Therefore, $0.1 \mathrm{mM}$ was selected as the optimal concentration of the MeSA 20 EC formulation for effectively controlling PWD.

The optimal treatment time was determined using $0.1 \mathrm{mM}$ MeSA $20 \mathrm{EC}$ as the optimal concentration. The control values were 48.9 and $40.0 \%$ for T2 and T3, respectively. There was no significant difference between T2 and T3, but considering T4 (4.4\%), we inferred that longer treatment interval worsened the efficacy. Therefore, the optimal treatment time was selected as T2 (Figure 3B).

\section{Evaluation of Disease Control Efficacy of Methyl Salicylate 20 DC Against Pine Wilt Disease Under Field Conditions}

The disease control efficacy of MeSA 20 DC formulation was evaluated under field conditions using 10-year-old $P$. densiflora. The disease incidences in response to treatment with MeSA 20 DC and control were 25 and $62.5 \%$, respectively, at 4 months post-inoculation (Figure 4A). In addition, MeSA $20 \mathrm{DC}$ and emamectin benzoate $2.15 \mathrm{EC}$, which was used as positive controls, showed control values of 60 and $46.7 \%$, respectively, compared to the untreated control. There was no significant difference between MeSA and emamectin benzoate (Figure 4B). The pictures taken using drone showed distinct wilting brownish between MeSA treatment and untreated control (Figure 4C). Therefore, based on the results of the field tests, we confirmed that MeSA can effectively control PWD in adult trees as well as 3-year-old seedlings.

\section{Assessment of the Effect of Methyl Salicylate on the Expression of Defense-Related Genes Using qRT-PCR}

The expression of defense-related genes was analyzed using qRT-PCR following treatment with PWN, MeSA, and both PWN and MeSA (Figure 5A). Expressions of $P R-1, P R-2, P R-$ 5, and ETS were modulated upon PWN inoculation or MeSA spraying. The relative expression level of the $P R-1$ was similar to or slightly higher in the MeSA-treated group than in the untreated group; however, the MeSA-treated group showed a 2.2-fold higher expression level than the untreated group at 7 days post-inoculation with PWN (I7) (Figure 5B). The relative 


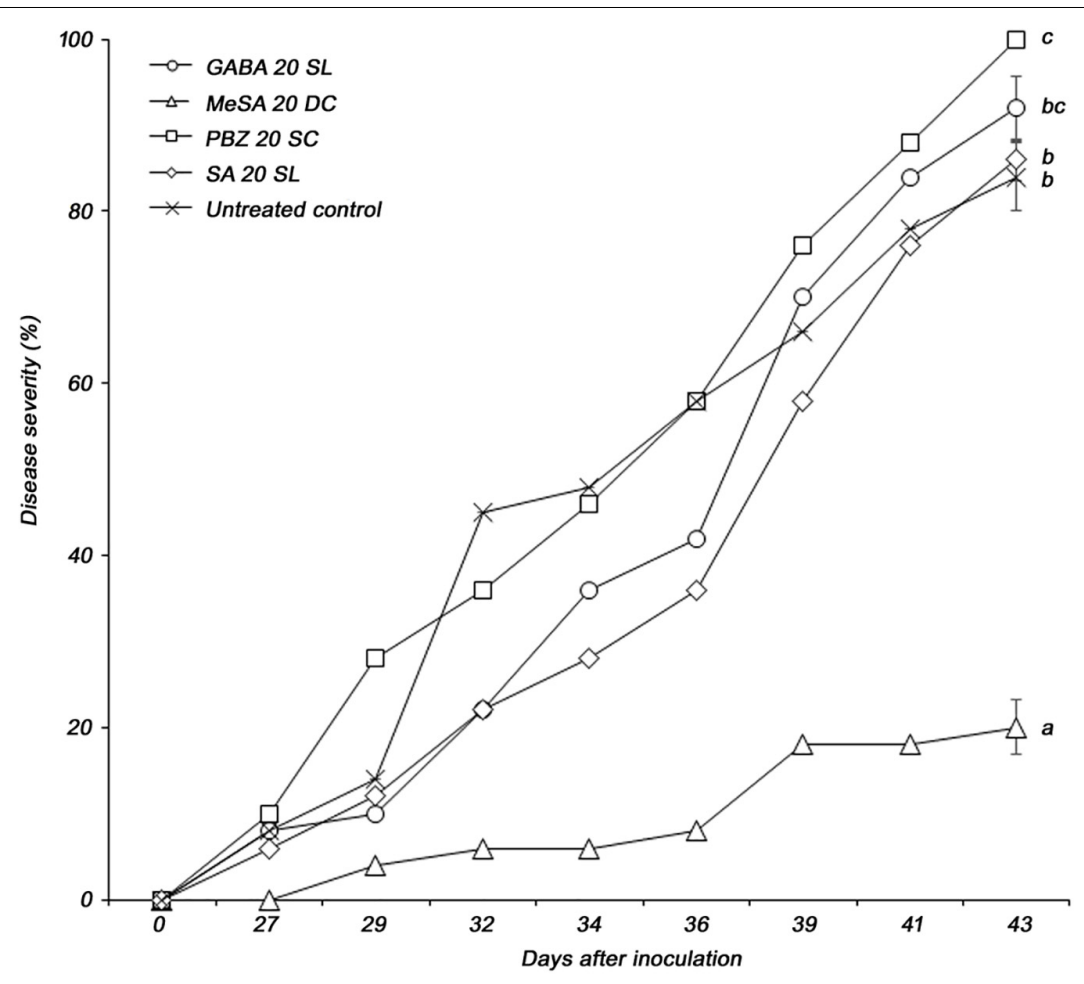

FIGURE 1 | Pine wilt disease severity in 3-year-old Pinus densiflora seedlings treated by foliar application with formulations of four elicitors (GABA, $\gamma$-aminobutyric acid; MeSA, methyl salicylate; PBZ, probenazole; and SA, salicylic acid). Each value represents the mean \pm standard error of five replicates. The different letters indicate significant differences with $p<0.05$, as calculated using the Tukey's HSD test.

expression level of the $P R-2$ was 6.2- and 5.7-fold higher than that of the untreated control group at 7 days post-first treatment (1T7) and 3 days post-second treatment with MeSA (2T3), respectively. Post-inoculation with $\mathrm{PWN}$, the expression of $P R$ 2 was consistently higher than that of the untreated group. The expression levels of $P R-2$ in the untreated group also increased over time, but the MeSA-treated group showed a much greater increase; in particular, it displayed 4.4- and 4.5-fold higher upregulation than the untreated group at 3 and 7 days postinoculation with PWN (I3 and I7), respectively (Figure 5C). The relative expression levels of $P R-5$ was 3.1- and 6-fold higher in the MeSA-treated group than in the untreated group at 7 days post-first and -second treatment with MeSA (1T7 and 2T7), and 1.8- and 2.2-fold higher, respectively, at 3 and 7 days post-inoculation with PWN (Figure 5D). $P R-1, P R-2$, and $P R-5$, which are the markers of salicylic acid-dependent SAR, showed similar patterns of upregulation as the untreated groups, posttreatment with MeSA and -PWN inoculation. Upon treatment with PWN or MeSA, PR-9 expression was induced 5- or 2-fold, respectively, as compared to the untreated condition, at all the time points monitored. Upon treatment with both PWN and MeSA, however, PR-9 expression was induced up to 15 -fold as compared to that in the normal condition, and 2-3-fold that in the PWN inoculation only condition (Figure 5E). These results demonstrated the boosting effect of MeSA treatment, presenting a strong evidence that MeSA treatment and PWN target the same plant defense system.
The MeSA-treated group displayed 2.5-fold higher expression of ETS, a cell wall-related gene, than the untreated group, at 3 days post-second treatment with MeSA (2T3). The expression level of ETS markedly increased 17.1- and 5.1-fold, respectively, compared to the untreated group, at 3 and 7 days postinoculation with PWN (I3 and I7) (Figure 5F). Similarly, the PR-9 expression was also induced at I3 and I7, as compared to the untreated group (2.7- and 2.1-fold, respectively). On the contrary, expression of XET, which loosens the cell wall, was downregulated as compared to the untreated group, except at 1 day post-first treatment with MeSA, and was expressed at a very low level post-inoculation with PWN (Figure 5G).

Based on the results of the gene expression test, we concluded that treatment with MeSA via foliar spraying induced SAR in pine trees, including the PWN-related defense system. In addition, MeSA application strengthened the cell wall structure by upregulating ETS or downregulating XET expression, thereby increasing pine tree defense against PWN.

\section{DISCUSSION}

The control of PWD is a challenging process particularly in large scale forests where trunk injection with nematicides is hardly applicable and would require rigorous effort. In addition, spraying insecticides against the Monochamus vector, that is among the main methods used for conventional PWD 


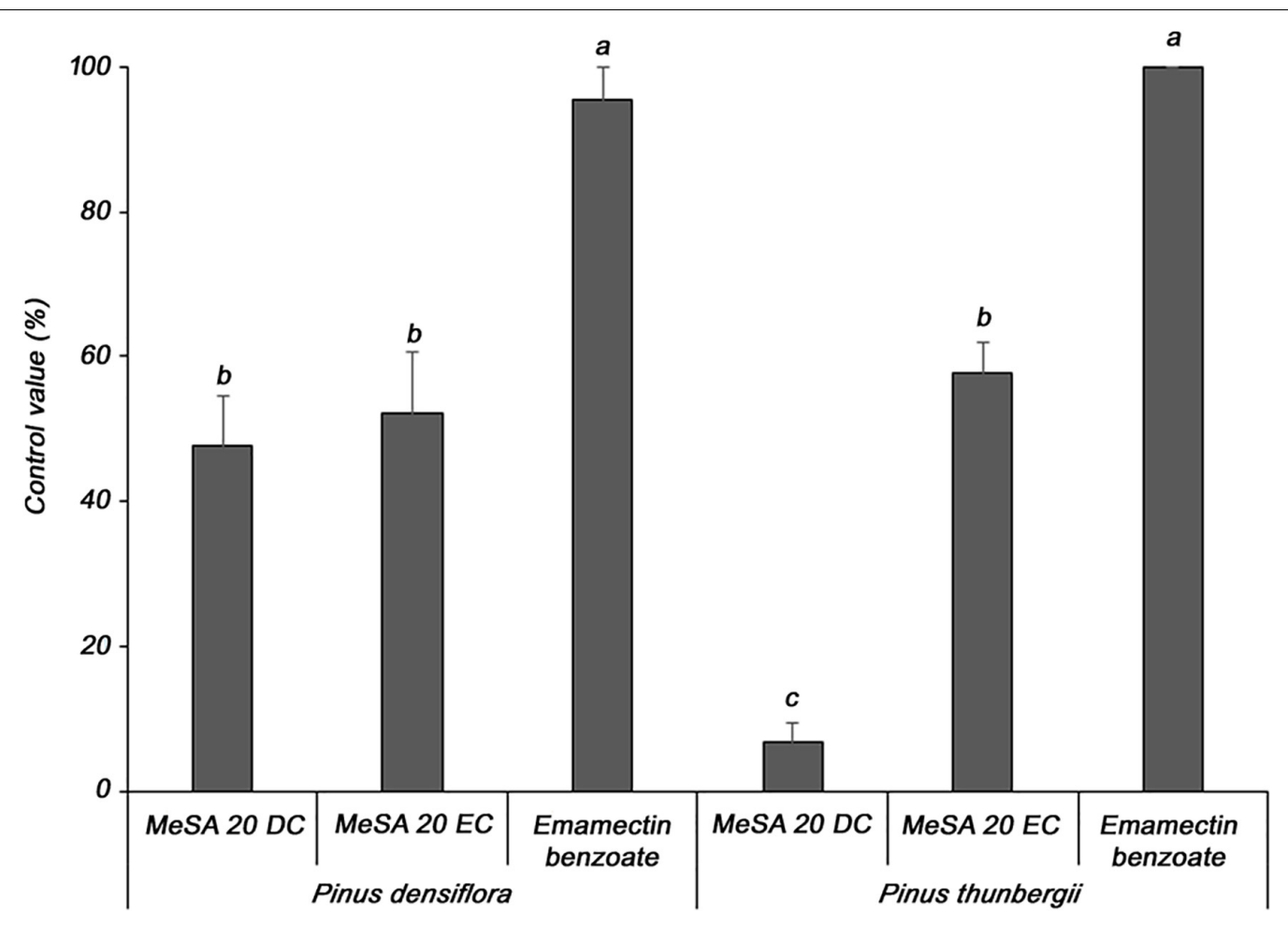

FIGURE 2 | Determination of the optimal formulation of methyl salicylate against pine wilt disease in 3-year-old Pinus densiflora and Pinus thunbergii seedlings. The formulations of methyl salicylate were treated with $10 \mathrm{mM}$, and emamectin benzoate was treated with $20 \mathrm{mg} / \mathrm{mL}$. Each value represents the mean \pm standard error of five replicates. Different letters indicate significant differences with $p<0.05$, as calculated using the Tukey's HSD test.

management, was shown to have a limited effect in hindering PWD spread and might result in emergence of insect resistance along with encompassing a great deal of environmental burden and adverse effects on human and other non-target species (Ikenaka et al., 2019; Jung et al., 2021). Induction of resistance using chemical elicitors represent a promising alternative that is sustainable and eco-friendly as there is no direct nematicidal activity or effect on other non-target species (Oka et al., 2000). However, utilization of induced resistance elicitors require evaluation and accurate setting up of the effective formulation, concentration, and time of foliar spray application. In this study, four SAR elicitors, including MeSA, were selected among 12 candidates known to induce resistance in various plants, based on in vivo screening via trunk injection into 3-yearold $P$. densiflora seedlings. The stability and effectiveness of the four selected resistance elicitors were assessed and MeSA was selected post-in vivo testing of its potency upon application as a foliar spray. MeSA is a volatile long-range signaling compound produced by many plants for induction of SAR and may activate resistance in neighboring plants or in the healthy tissues of the infected plant. MeSA in the air is absorbed by the uninfected distal tissue and converted to SA for the establishment of acquired resistance (Shulaev et al., 1997). Signaling in plants by volatile compounds such as MeSA can overcome the spatial and temporal limitations of the vascular system (Heil and Ton, 2008). The timing of defense responses is crucial and can mark the difference between whether the plant overcomes or succumbs to the pathogen infection.

Two types of MeSA formulations (MeSA 20 DC and MeSA 20 EC) were tested for foliar spray application in this study. Unlike $P$. densiflora, treatment with MeSA 20 DC was ineffective in controlling PWD when tested on $P$. thunbergii seedlings. Although both $P$. densiflora and $P$. thunbergii belong to the same genus Pinus, their leaves are histologically different, as P. thunbergii has a thicker epidermis and stronger subcutaneous tissue than P. densiflora (Shaw, 1914). To overcome this difference, a new formulation, MeSA 20 EC, was prepared. Upon comparing the two formulations, MeSA 20 EC showed better control efficiency in both $P$. densiflora and $P$. thunbergii seedlings (52.3 and 57.8\%, respectively), while MeSA 20 DC showed a similar effect $(47.7 \%)$ to MeSA 20 EC in P. densiflora, but not in P. thunbergii (6.7\%). Unlike DC, EC uses methylated soy bean oil (MSO2) as the solvent with a high flash point, thus resulting in a lower volatility of MeSA, allowing for prolonged exposure on the leaves, thus improving its efficiency on $P$. thunbergii. Therefore, the MeSA 20 EC formulation was selected as the optimal formulation.

Resistance inducing elicitors generally show maximum efficiency at specific concentrations, depending on the plant, unlike chemicals that have direct concentrationdependent antimicrobial activity. For example, SA showed the highest effect at a concentration of $1.5 \mathrm{mM}$ against Fusarium oxysporum in tomato and at a concentration of 
A

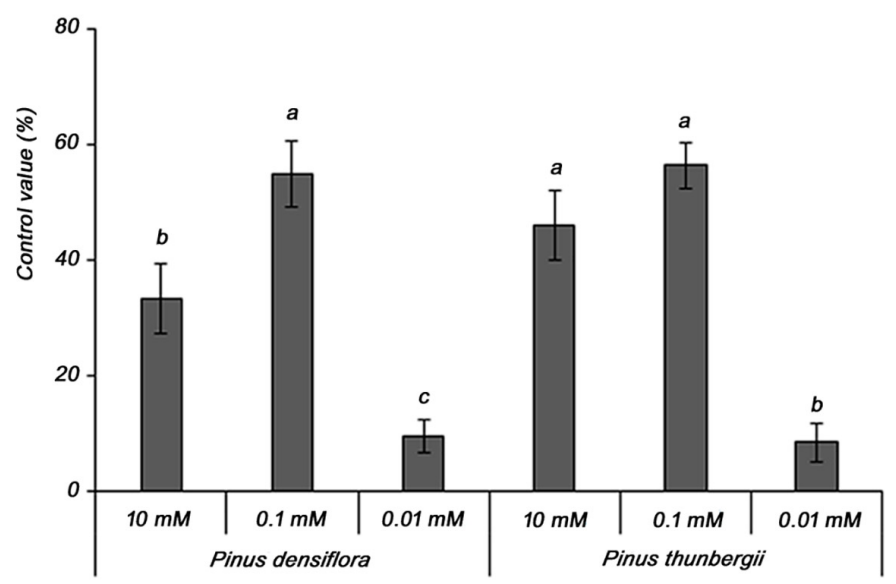

B

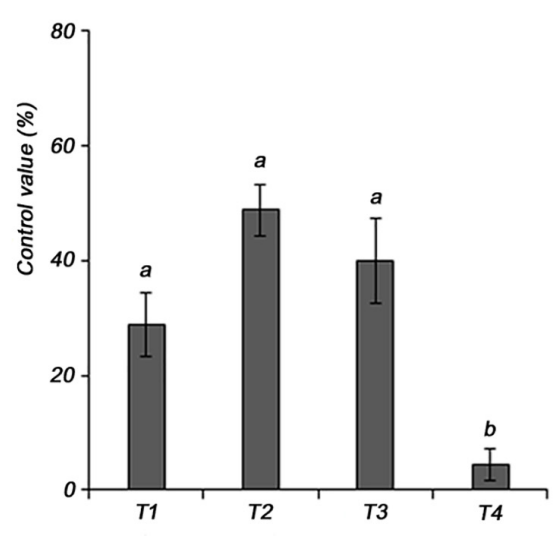

C

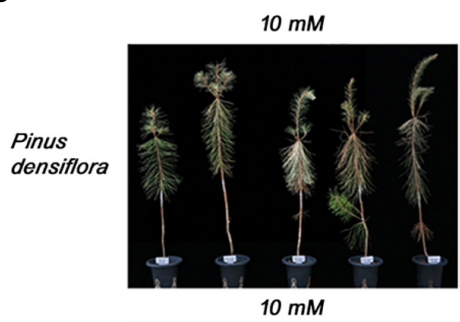

$10 \mathrm{mM}$

$0.1 \mathrm{mM}$

$0.01 \mathrm{mM}$

Untreated control
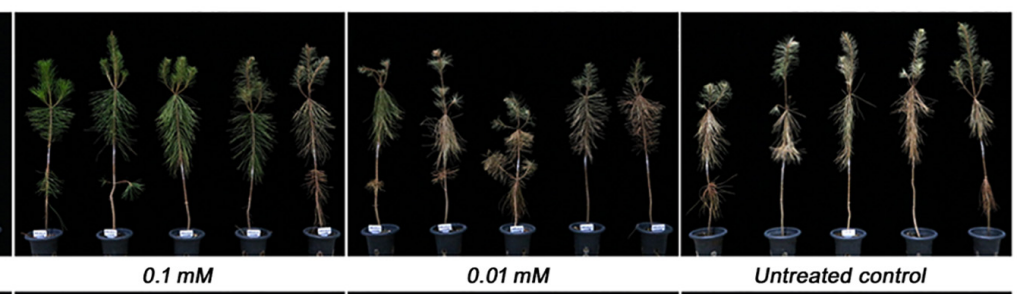

Untreated control
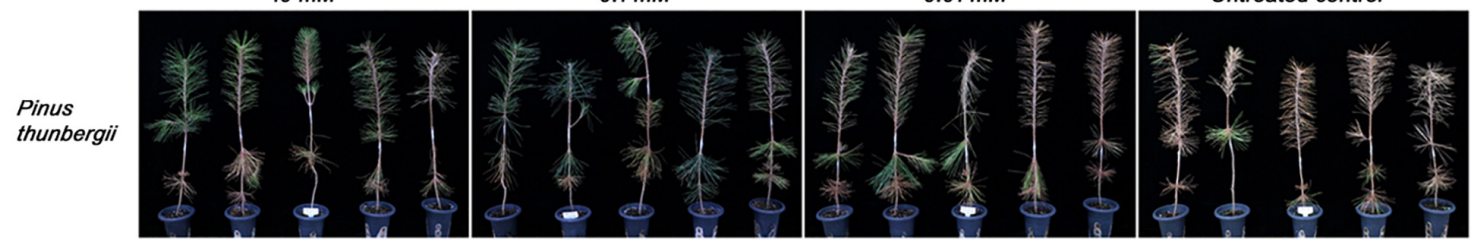

FIGURE 3 | Determination of (A) optimal concentration of methyl salicylate 20 EC in 3-year-old Pinus densiflora and Pinus thunbergii seedlings, and (B) application time of $0.1 \mathrm{mM}$ methyl salicylate $20 \mathrm{EC}$ in 3-year-old Pinus densiflora seedlings against pine wilt disease. T1, treatment at 1 week before inoculation with pine wood nematode; T2, treatment at 2 and 1 weeks before inoculation with pine wood nematode; T3, treatment at 3 and 1 weeks before inoculation with pine wood nematode; T4, treatment at 4 and 1 week before inoculation with pine wood nematode. Each value represents the mean \pm standard error of five replicates. Different letters indicate significant differences with $p<0.05$, as calculated using the Tukey's HSD test. (C) The representing pictures of $P$. densiflora and $P$. thunbergii after inoculation with PWN with or without MeSA. From 10 to $0.01 \mathrm{mM}$ of MeSA was treated 2 and 1 weeks before PWN inoculation with indicated concentrations.

$0.5 \mathrm{mM}$ against Ralstonia solanacearum in pepper (Ojha and Chatterjee, 2012; Chandrasekhar et al., 2017). We tested several concentrations of MeSA 20 EC formulation to determine the optimal concentration, which was eventually selected as the optimal formulation for treating PWD. The highest control efficiency was observed at MeSA concentration of $0.1 \mathrm{mM}$ in both P. densiflora and P. thunbergii (54.8 and 56.3\%, respectively). Using this optimum concentration of MeSA 20 EC, the optimal treatment time was tested and confirmed the control values of 48.9 and $40.0 \%$ at $\mathrm{T} 2$ and $\mathrm{T} 3$, respectively. There was no significant difference between $\mathrm{T} 2$ and $\mathrm{T} 3$, but considering the effect at T4 (4.4\%), we inferred that the longer treatment interval worsened the efficacy; thus, T2 was selected as the optimal treatment time. Based on these results, it was established that treatment with MeSA 20 EC formulation at a concentration of $0.1 \mathrm{mM}$ at 2 and 1 week before inoculation of PWN (T2) were the optimal formulation and processing conditions for MeSA, respectively, to achieve effective control against PWD.
Field experiments on 10-year-old $P$. densiflora have also confirmed the efficiency of MeSA foliar treatment against PWD as the disease incidence of treated trees were significantly reduced to levels comparable to those achieved with trunk injection with Emamectin benzoate. However, since we have established that $0.1 \mathrm{mM}$ of MeSA EC is the optimal treatment condition through the optimized greenhouse test of MeSA, additional field tests according to the optimal treatment conditions are required, and a better effect can be expected.

Our previous studies reported genome-wide analyses of pine trees treated with MeSA against the nematode B. xylophilus (Park et al., 2020). The application of the MeSA, effective in terms of reducing PWD severity, mainly induce genes involved in systems for protection from ROS damage as well as genes encoding flavonoid biosynthesis. Flavonoids not only contribute to a neutralizing ROS (Agati et al., 2012), but affect the fitness of nematodes at different life stages (Chin et al., 2018) based on network and gene ontology (GO) analyze. In this study, 
A

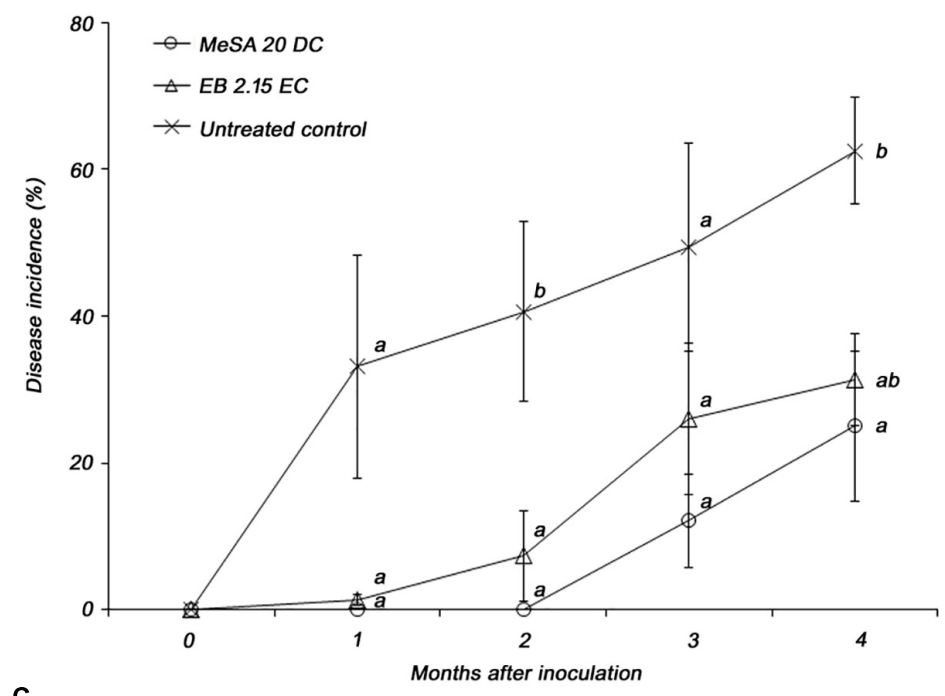

C

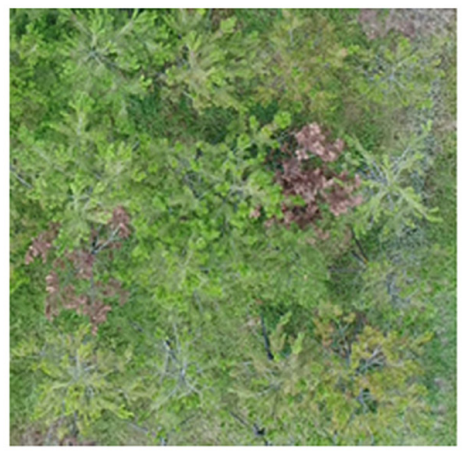

MeSA $20 D C$

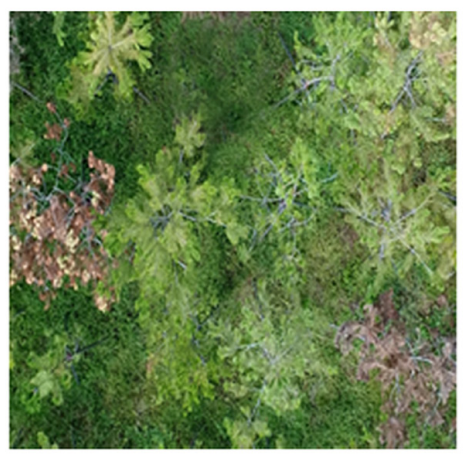

$E B$ 2.15 EC
B
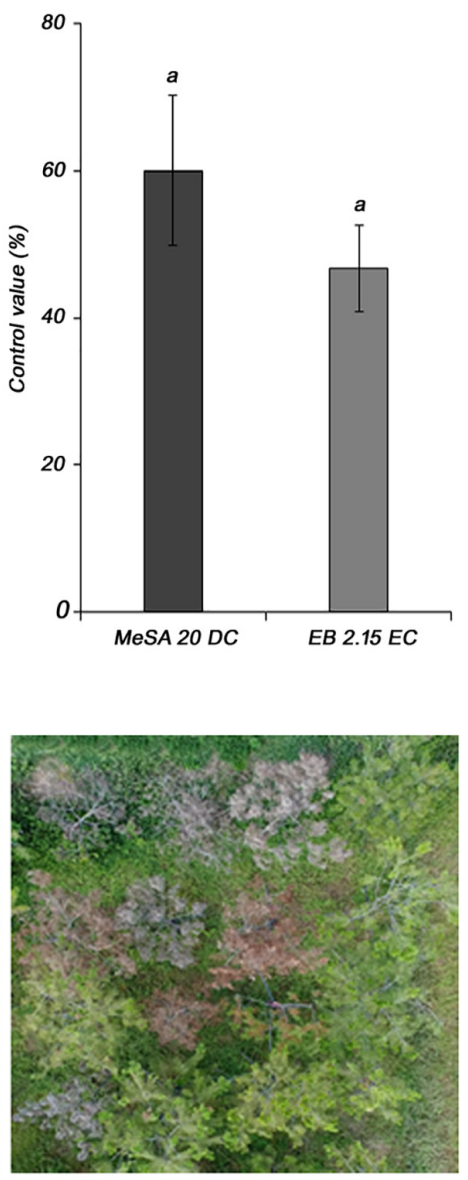

Untreated control

FIGURE 4 | Evaluation of disease control efficacy of methyl salicylate 20 DC (MeSA 20 DC) against pine wilt disease upon foliar application onto 10-year-old Pinus densiflora under field conditions. Emamectin benzoate 2.15 EC (EB 2.15 EC) was used as positive control and applied via trunk injection. Each pine tree was inoculated with 10,000 juveniles of pine wood nematode. (A) Disease severity after inoculation of pine wood nematode. Each value represents the mean \pm standard error of four replicates with each replicate consist of four trees. The different letters indicate significant differences with $p<0.05$, as calculated using the Tukey's HSD test. (B) Disease control efficacy at 4 months post-inoculation with pinewood nematode. (C) The pictures of pine trees treated with MeSA 20 DC, EB 2.15 EC, and Tween ${ }^{\circledR} 20$ (untreated control).

we focused on expression analyze of defense-related genes. It has been reported that the transcript levels of defenserelated gene expression are different between susceptible and resistance pine trees and the inoculation with PWN further increase such difference in gene expression levels (Hirao et al., 2012). To elucidate the mechanism underlying the observed control value of MeSA against PWD, qRT-PCR analysis was performed in this study using $P$. densiflora leaves. When MeSA was applied to pine leaves, $P R-1, P R-2$, and $P R-5$ expressions were significantly upregulated compared to the untreated group, before and after inoculation with PWN. $P R-1, P R-2$, and $P R-5$ are commonly used as marker genes for SAR induction (Molinari et al., 2014). Recent studies have reported that upregulation of $P R-1, P R-2$, and $P R-5$ expression significantly inhibits infection of beet-cyst (Heterodera schachtii) and rootknot (Meloidogyne incognita) nematodes in Arabidopsis thaliana and tomato (Uehara et al., 2010; Hamamouch et al., 2011).
Furthermore, it has been reported that the difference between whether a plant is susceptible or resistant depends on the differences in the timing and magnitude of defense response (Tao et al., 2003). Therefore, the optimum timing and high expression of the $P R$ may contribute to the effective inhibition of PWD development.

Upon MeSA treatment, expressions of cell wall-related genes, PR-9 and ETS, were upregulated, while that of XET was downregulated. Extensin, encoded by the ETS, is believed to play an important part in cell wall biosynthesis in plants (Cannon et al., 2008). Extensin plays an essential role in biotic and abiotic stress responses by being involved in maintaining the strength of the cell wall (Deepak et al., 2010). In addition, peroxidase encoded by the $P R-9$ strengthens the cell wall by catalyzing lignification and enhancing resistance against various pathogens (Van Loon et al., 2006). The XET regulates the strength of the cell wall by being involved in xyloglucan, which supports the load between 
A

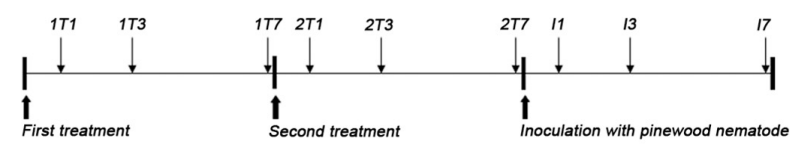

B

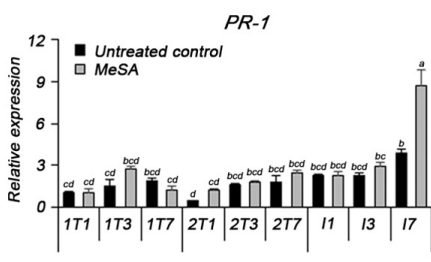

C

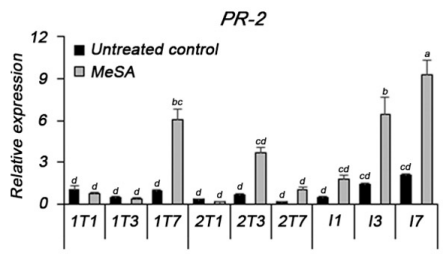

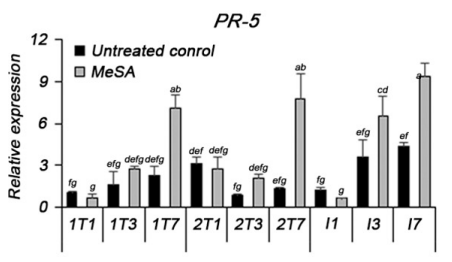

G

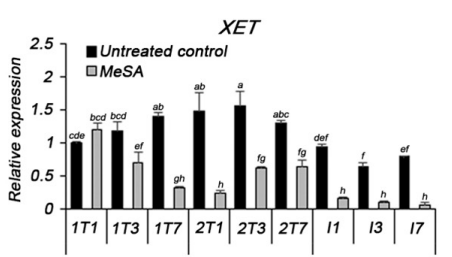

FIGURE 5 | (A) The plan of methyl salicylate treatment, pinewood nematode inoculation, and sampling for qRT-PCR analysis. qRT-PCR analysis of transcripts of (B) pathogenesis-related protein 1 (PR-1), (C) pathogenesis-related protein 2 (PR-2), (D) pathogenesis-related protein 5 (PR-5), (E) pathogenesis-related protein 9 (PR-9), (F) extensin (ETS), and (G) xyloglucan endotransglycosylase (XET).

the cellulose microfibers in the cell wall (Uozu et al., 2000; Vissenberg et al., 2000). Strengthening of the cell wall is a very important factor during the early stages of plant defense. In a study comparing histological changes in susceptible and resistant $P$. thunbergii, the resistant $P$. thunbergii effectively inhibited the initial migration of PWN by strengthening the cell wall via lignification (Kusumoto et al., 2014). In addition, migration and reproduction of PWN are important factors that determine the survival of pine trees (Nakajima et al., 2019). Therefore, MeSA is believed to be involved in a control mechanism against PWD by rapidly inducing the expression of defense-related genes in pine trees prior to infection, promptly responding to invasion of PWN, and delaying migration and reproduction of PWN.

Overall, this study provides evidence on the effective control activity of foliar spray with MeSA against PWD under greenhouse and field conditions as possible alternative to other pesticide control methods. The mechanism of control was indicated as induction of resistance by the observed significant changes in the expression of defense-related genes without direct nematicidal activity. The optimum formulation, concentration and treatment time were adjusted for improvement of the control activity. The provided results represent an important step toward the establishment of effective eco-friendly and applicable control method for PWD under field conditions.

\section{CONCLUSION}

MeSA was selected from 12 candidate elicitors via in vivo screening using P. densiflora seedlings. MeSA 20 DC formulation showed a control efficacy of $60 \%$ in field tests using 10 year-old $P$. densiflora. The optimal formulation and processing conditions for treatment were established as $0.1 \mathrm{mM}$ MeSA $20 \mathrm{EC}$ at 2 and 1 WBI with PWN. In addition, qRTPCR analysis showed that MeSA induced elevated expression of various defense-related genes in pine leaves. The high expression of various plant defense-related and cell wall-related genes suggests that PWD can be controlled by inhibiting the migration and reproduction of PWN. Collectively, these results suggest that foliar application of MeSA may reduce the incidence of PWD by inducing resistance, thereby providing an economically feasible alternative to trunk-injection agents for the management of PWD.

\section{DATA AVAILABILITY STATEMENT}

The original contributions presented in the study are included in the article/Supplementary Material, further inquiries can be directed to the corresponding author/s.

\section{AUTHOR CONTRIBUTIONS}

Y-SS and J-CK conceived the study. HJ, AP, MS, NK, GH, and JK performed the experiments. HJ, AP, Y-SS, and J-CK analyzed the data. HJ wrote the manuscript. AP, Y-SS, YK, and J-CK reviewed and edited the manuscript. J-CK acquired the funds. All authors have read and agreed to the published version of the manuscript. 


\section{FUNDING}

This research was supported by a grant funded by National Institute of Forest Science, South Korea (FE0702-2016-02).

\section{ACKNOWLEDGMENTS}

The pinewood nematode, Bursaphelenchus xylophilus, used in this experiment was kindly provided by Dr. Kim Junheon,

\section{REFERENCES}

Abbott, W. S. (1925). A method of computing the effectiveness of an insecticide. J. Econ. Entomol. 18, 265-267. doi: 10.1093/jee/18.2.265a

Abelleira, A., Picoaga, A., Mansilla, J., and Aguin, O. (2011). Detection of Bursaphelenchus xylophilus, causal agent of pine wilt disease on Pinus pinaster in Northwestern Spain. Plant Dis. 95, 776-776. doi: 10.1094/PDIS-12-1 0-0902

Agati, G., Azzarello, E., Pollastri, S., and Tattini, M. (2012). Flavonoids as antioxidants in plants: location and functional significance. Plant Sci. 196, 67-76. doi: 10.1016/j.plantsci.2012.07.014

Azevedo, H., Lino-Neto, T., and Tavares, R. M. (2003). An improved method for high-quality RNA isolation from needles of adult maritime pine trees. Plant Mol. Biol. Rep. 21, 333-338. doi: 10.1007/bf02772582

Baermann, G. (1917). Eine einfache methode zur auffindung von Ancylostomum (Nematoden) larven in erdproben. Geneeskd. Tijdschr. Ned. Indie. 57, 131-137.

Cannon, M. C., Terneus, K., Hall, Q., Tan, L., Wang, Y., Wegenhart, B. L., et al. (2008). Self-assembly of the plant cell wall requires an extensin scaffold. Proc. Natl. Acad. Sci. 105, 2226-2231. doi: 10.1073/pnas.0711980105

Chandrasekhar, B., Umesha, S., and Kumar, H. N. (2017). Proteomic analysis of salicylic acid enhanced disease resistance in bacterial wilt affected chilli (Capsicum annuum) crop. Physiol. Mol. Plant Pathol. 98, 85-96. doi: 10.1016/j. pmpp.2017.04.002

Cheng, H., Lin, M., Li, W., and Fang, Z. (1983). The occurrence of a pine wilting disease caused by a nematode found in Nanjing. For. Pest Dis. 4, 1-5.

Chin, S., Behm, C. A., and Mathesius, U. (2018). Functions of flavonoids in plant-nematode interactions. Plants 7:85. doi: 10.3390/plants7040085

Deepak, S., Shailasree, S., Kini, R. K., Muck, A., Mithöfer, A., and Shetty, S. H. (2010). Hydroxyproline-rich glycoproteins and plant defence. J. Phytopathol. 158, 585-593. doi: 10.1111/j.1439-0434.2010.01669.x

Dempsey, D. M. A., and Klessig, D. F. (2012). SOS-too many signals for systemic acquired resistance? Trends Plant Sci. 17, 538-545. doi: 10.1016/j.tplants.2012. 05.011

García-Pastor, M. E., Giménez, M. J., Zapata, P. J., Guillén, F., Valverde, J. M., Serrano, M., et al. (2020). Preharvest application of methyl salicylate, acetyl salicylic acid and salicylic acid alleviated disease caused by Botrytis cinerea through stimulation of antioxidant system in table grapes. Int. J. Food Microbiol. 334:108807. doi: 10.1016/j.ijfoodmicro.2020.108807

Hamamouch, N., Li, C., Seo, P. J., Park, C. M., and Davis, E. L. (2011). Expression of Arabidopsis pathogenesis-related genes during nematode infection. Mol. Plant Pathol. 12, 355-364. doi: 10.1111/j.1364-3703.2010.00675.x

Hayat, S., and Ahmad, A. (2007). Salicylic acid-a plant hormone. New York,NY: Springer Science \& Business Media.

Heil, M., and Ton, J. (2008). Long-distance signalling in plant defence. Trends Plant Sci. 13, 264-272. doi: 10.1016/j.tplants.2008.03.005

Hirao, T., Fukatsu, E., and Watanabe, A. (2012). Characterization of resistance to pine wood nematode infection in Pinus thunbergii using suppression subtractive hybridization. BMC Plant Biol. 12:1-14. doi: 10.1186/1471-2229$12-13$

Ikenaka, Y., Miyabara, Y., Ichise, T., Nakayama, S., Nimako, C., Ishizuka, M., et al. (2019). Exposures of children to neonicotinoids in pine wilt disease control areas. Environ. Toxicol. Chem. 38, 71-79. doi: 10.1002/etc.4316

Ji, H., Kyndt, T., He, W., Vanholme, B., and Gheysen, G. (2015). $\beta$-Aminobutyric acid-induced resistance against root-knot nematodes in rice is based on
National Institute of Forest Science, South Korea. We would like to thank the director of Yoosung Chemical R\&T Co., Ltd., Mr. Moon Sung Kang, for his help in preparing the formulations.

\section{SUPPLEMENTARY MATERIAL}

The Supplementary Material for this article can be found online at: https://www.frontiersin.org/articles/10.3389/fpls.2021. 812414/full\#supplementary-material

increased basal defense. Mol. Plant Microbe Interact. 28, 519-533. doi: 10.1094/ MPMI-09-14-0260-R

Jung, H. W., Tschaplinski, T. J., Wang, L., Glazebrook, J., and Greenberg, J. T. (2009). Priming in systemic plant immunity. Science 324, 89-91. doi: 10.1126/ science. 1170025

Jung, J. K., Lee, U. G., Cha, D., Kim, D. S., and Jung, C. (2021). Can insecticide applications used to kill vector insects prevent pine wilt disease? Pest Manag. Sci. 77, 4923-4929. doi: 10.1002/ps.6532

Kalaivani, K., Kalaiselvi, M. M., and Senthil-Nathan, S. (2016). Effect of methyl salicylate (MeSA), an elicitor on growth, physiology and pathology of resistant and susceptible rice varieties. Sci. Rep. 6, 1-11. doi: 10.1038/srep34498

Kim, J., Jung, Y. H., and Lee, S.-M. (2021). Diel Rhythmicity of Field Responses to Synthetic Pheromone Lures in the Pine Sawyer Monochamus saltuarius. Insects 12:441. doi: 10.3390/insects 12050441

Kim, J., Seo, S.-M., and Park, I.-K. (2011). Nematicidal activity of plant essential oils and components from Gaultheria fragrantissima and Zanthoxylum alatum against the pine wood nematode. Bursaphelenchus xylophilus. Nematology 13, 87-93. doi: 10.1163/138855410x504907

Kim, N., Jeon, H. W., Mannaa, M., Jeong, S. I., Kim, J., Kim, J., et al. (2019). Induction of resistance against pine wilt disease caused by Bursaphelenchus xylophilus using selected pine endophytic bacteria. Plant Pathol. 68, 434-444. doi: $10.1111 /$ ppa. 12960

Korea Forest Service (2013). Guideline for the control of forest diseases and insect pests. Dunsan-dong: Korea Forest Service, Daejeon, Republic of Korea, 325.

Kusumoto, D., Yonemichi, T., Inoue, H., Hirao, T., Watanabe, A., and Yamada, T. (2014). Comparison of histological responses and tissue damage expansion between resistant and susceptible Pinus thunbergii infected with pine wood nematode Bursaphelenchus xylophilus. J. For. Res. 19, 285-294. doi: 10.1007/ s10310-013-0417-y

Kwon, H. R., Choi, G. J., Choi, Y. H., Jang, K. S., Sung, N. D., Kang, M. S., et al. (2010). Suppression of pine wilt disease by an antibacterial agent, oxolinic acid. Pest Manag. Sci. 66, 634-639. doi: 10.1002/ps.1920

Kwon, T.-S., Song, M.-Y., Shin, S.-C., and Park, Y.-S. (2005). Effects of aerial insecticide sprays on ant communities to control pine wilt disease in Korean pine forests. Appl. Entomol. Zool. 40, 563-574. doi: 10.1303/aez.2005.563

Lee, I. H., Han, H., Koh, Y. H., Kim, I. S., Lee, S.-W., and Shim, D. (2019). Comparative transcriptome analysis of Pinus densiflora following inoculation with pathogenic (Bursaphelenchus xylophilus) or non-pathogenic nematodes (B. thailandae). Sci. Rep. 9, 1-11. doi: 10.1038/s41598-019-48660-w

Lee, S.-M., Kim, D.-S., Lee, S.-G., Park, N.-C., and Lee, D.-W. (2009). Selection of trunk injection pesticides for preventive of pine wilt disease, Bursaphelenchus xylophilus on Japanese black pine (Pinus thunbergii). Korean J. Pestic Sci. 13, 267-274.

Li, M., Li, H., Sheng, R.-C., Sun, H., Sun, S.-H., and Chen, F.-M. (2020). The first record of Monochamus saltuarius (Coleoptera; Cerambycidae) as vector of Bursaphelenchus xylophilus and its new potential hosts in China. Insects 11:636. doi: 10.3390/insects11090636

Livak, K. J., and Schmittgen, T. D. (2001). Analysis of relative gene expression data using real-time quantitative PCR and the $2^{-\Delta \Delta}$ CT method. Methods 25, 402-408. doi: 10.1006/meth.2001.1262

Maehara, N., and Futai, K. (2000). Population changes of the pinewood nematode, Bursaphelenchus xylophilus (Nematoda: Aphelenchoididae), on fungi growing in pine-branch segments. Appl. Entomol. Zool. 35, 413-417. doi: 10.1303/aez.20 00.413 
Mamiya, Y. (1988). History of pine wilt disease in Japan. J. Nematol. 20:219.

Molinari, S., Fanelli, E., and Leonetti, P. (2014). Expression of tomato salicylic acid (SA)-responsive pathogenesis-related genes in $M i-1-$ mediated and SA-induced resistance to root-knot nematodes. Mol. Plant Pathol. 15, 255264. doi: $10.1111 / \mathrm{mpp} .12085$

Mota, M., Burgermeister, W., Braasch, H., Sousa, E., Penas, A. C., Metge, K., et al. (1999). First report of Bursaphelenchus xylophilus in Portugal and in Europe. Nematology 1, 727-734. doi: 10.1163/156854199508757

Nakajima, G., Iki, T., Yamanobe, T., Nakamura, K., and Aikawa, T. (2019). Spatial and temporal distribution of Bursaphelenchus xylophilus inoculated in grafts of a resistant clone of Pinus thunbergii. J. For. Res. 24, 93-99. doi: 10.1080/ 13416979.2019.1578136

Ojha, S., and Chatterjee, N. C. (2012). Induction of resistance in tomato plants against Fusarium oxysporum f. sp. lycopersici mediated through salicylic acid and Trichoderma harzianum. J. Plant Prot. Res. 52:3. doi: 10.2478/v10045-0120034-3

Oka, Y., Koltai, H., Bar-Eyal, M., Mor, M., Sharon, E., Chet, I., et al. (2000). New strategies for the control of plant-parasitic nematodes. Pest. Manag. Sci. 56, 983-988. doi: 10.1002/1526-4998(200011)56:11<983::AID-PS233<3.0.CO;2-X

Park, J., Jeon, H. W., Jung, H., Lee, H. H., Kim, J., Park, A. R., et al. (2020). Comparative Transcriptome Analysis of Pine Trees Treated with ResistanceInducing Substances against the Nematode Bursaphelenchus xylophilus. Genes 11:1000. doi: 10.3390/genes11091000

Park, S.-W., Kaimoyo, E., Kumar, D., Mosher, S., and Klessig, D. F. (2007). Methyl salicylate is a critical mobile signal for plant systemic acquired resistance. Science 318, 113-116. doi: 10.1126/science.1147113

Proença, D. N., Francisco, R., Santos, C. V., Lopes, A., Fonseca, L., Abrantes, I. M., et al. (2010). Diversity of bacteria associated with Bursaphelenchus xylophilus and other nematodes isolated from Pinus pinaster trees with pine wilt disease. PLoS One. 5:e15191. doi: 10.1371/journal.pone.0015191

Shaw, G. R. (1914). The genus Pinus. Riverside: Riverside Press.

Shulaev, V., Silverman, P., and Raskin, I. (1997). Airborne signalling by methyl salicylate in plant pathogen resistance. Nature 385, 718-721. doi: 10.1038/ $385718 \mathrm{a} 0$

Spoel, S. H., and Dong, X. (2012). How do plants achieve immunity? Defence without specialized immune cells. Nat. Rev. Immunol. 12, 89-100. doi: 10.1038/ nri3141

Tao, Y., Xie, Z., Chen, W., Glazebrook, J., Chang, H.-S., Han, B., et al. (2003). Quantitative nature of Arabidopsis responses during compatible and incompatible interactions with the bacterial pathogen Pseudomonas syringae. Plant Cell 15, 317-330. doi: 10.1105/tpc.007591

Tzean, S., and Jan, S. (1985). "The occurrence of pine wood nematode, Bursaphelenchus xylophilus," in Proceedings of the 6th ROC symposium of electron microscopy, (Taiwan), 38-39.
Uehara, T., Sugiyama, S., Matsuura, H., Arie, T., and Masuta, C. (2010). Resistant and susceptible responses in tomato to cyst nematode are differentially regulated by salicylic acid. Plant Cell Physiol. 51, 1524-1536. doi: 10.1093/pcp/ pcq109

Uozu, S., Tanaka-Ueguchi, M., Kitano, H., Hattori, K., and Matsuoka, M. (2000). Characterization of XET-related genes of rice. Plant Physiol. 122, 853-860. doi: 10.1104/pp.122.3.853

Van Loon, L. C., Rep, M., and Pieterse, C. M. (2006). Significance of inducible defense-related proteins in infected plants. Annu. Rev. Phytopathol. 44, 135162. doi: 10.1146/annurev.phyto.44.070505.143425

Vissenberg, K., Martinez-Vilchez, I. M., Verbelen, J.-P., Miller, J. G., and Fry, S. C. (2000). In vivo colocalization of xyloglucan endotransglycosylase activity and its donor substrate in the elongation zone of Arabidopsis roots. Plant Cell 12, 1229-1237. doi: 10.2307/3871267

Vlot, A. C., Sales, J. H., Lenk, M., Bauer, K., Brambilla, A., Sommer, A., et al. (2021). Systemic propagation of immunity in plants. New Phytol. 229, 1234-1250. doi: $10.1111 / \mathrm{nph} .16953$

Yang, B., and Wang, Q. (1989). Distribution of the pinewood nematode in China and susceptibility of some Chinese and exotic pines to the nematode. Can. J. For. Res. 19, 1527-1530. doi: 10.1139/x89-232

Yano, S. (1913). Investigation on pine death in Nagasaki prefecture. SanrinKouhou. 4, 1-14.

Yi, C. K., Byun, B. H., Park, J. D., Yang, S., and Chang, K. H. (1989). First finding of the pine wood nematode, Bursaphelenchus xylophilus (Steiner et Buhrer) Nickle and its insect vector in Korea. Res. Rep. For. Res. Inst. Seoul. 38, 141-149.

Conflict of Interest: The authors declare that the research was conducted in the absence of any commercial or financial relationships that could be construed as a potential conflict of interest.

Publisher's Note: All claims expressed in this article are solely those of the authors and do not necessarily represent those of their affiliated organizations, or those of the publisher, the editors and the reviewers. Any product that may be evaluated in this article, or claim that may be made by its manufacturer, is not guaranteed or endorsed by the publisher.

Copyright (c) 2022 Jeon, Park, Sung, Kim, Mannaa, Han, Kim, Koo, Seo and Kim. This is an open-access article distributed under the terms of the Creative Commons Attribution License (CC BY). The use, distribution or reproduction in other forums is permitted, provided the original author(s) and the copyright owner(s) are credited and that the original publication in this journal is cited, in accordance with accepted academic practice. No use, distribution or reproduction is permitted which does not comply with these terms. 